\title{
Broad-spectrum Cross-resistance to Anticancer Drugs Mediated by Epidermal Growth Factor Receptor
}

\author{
GE YAN and THOMAS EFFERTH \\ Department of Pharmaceutical Biology, Institute of Pharmacy and Biochemistry, \\ Johannes Gutenberg University, Mainz, Germany
}

\begin{abstract}
Background: The oncogenic role of epidermal growth factor receptor (EGFR) has been intensively studied. However, its emerging role in drug resistance has not been fully addressed. Materials and Methods: This study systematically investigated the correlation of $m R N A$ and protein expression of EGFR, as well as gene amplification and mutations with the log-transformed half-maximal inhibitory concentration $\left(\log _{10} I_{50}\right)$ values obtained from the NCI panel of 60 human tumor cell lines against 83 standard anticancer agents and the top 10 natural cytotoxic products previously screened by us. Results: EGFR protein expression, rather than other measurements, was most frequently associated with drug response. $\log _{10} I_{50}$ and EGFR protein level were significantly positively correlated under all investigated DNA topoisomerase (TOPO) II inhibitors, followed by $81 \%$ of alkylating agents and platinum-based compounds, $71 \%$ of anti-hormones, $66 \%$ of TOPO I inhibitors and $50 \%$ of antibiotics. Furthermore, $60 \%$ of cytotoxic natural products did not reveal significant correlations. Conclusion: Collectively, we showed a broadspectrum of cross-resistance towards clinical drugs mediated by EGFR. Natural cytotoxic products may be further developed as novel drugs to overcome EGFR-associated resistance to clinically established anticancer drugs.
\end{abstract}

The epidermal growth factor receptor (EGFR) is an oncogenic receptor tyrosine kinase (RTK), which is commonly overexpressed or hyper-activated in solid tumors. Its downstream cascades upon activation are involved in carcinogenesis (1). Consequently, EGFR-driven therapeutic strategies have developed rapidly since the early 1990s (2).

Correspondence to: Professor Dr. T. Efferth, Department of Pharmaceutical Biology, Institute of Pharmacy and Biochemistry, Johannes Gutenberg University, Staudingerweg 5, 55128 Mainz, Germany. Tel: +49 61313925751, Fax: +49 61313923752, e-mail: efferth@uni-mainz.de

Key Words: Chemotherapy, oncogene, pharmacogenomics.
The tyrosine kinase activity of EGFR represents a successful therapeutic target. Three generations of tyrosine kinase inhibitors have been brought onto the market, e.g. gefitinib, afatinib and osimertinib. Despite the initial clinical benefit, many patients eventually experience disease progression due to mutation or alternative pathway activation $(3,4)$. Another pharmacological approach for impeding EGFR activation is the use of neutralizing monoclonal antibodies, e.g. cetuximab, panitumumab and nimotuzumab (5-7). These antibodies can induce antibody-dependent cellular cytotoxicity or complement-mediated cytotoxicity $(8,6)$. There are also studies demonstrating that these antibodies trigger internalization and thereafter degradation of EGFR, which further down-regulates the total EGFR level $(9,10)$.

In addition to its role as a prominent anticancer target, EGFR and its downstream signaling molecules are also considered to be emerging determinants of drug resistance to first-line chemotherapies and ironizing radiotherapy (11-13). Activation of EGFR-relevant molecules provide compensatory pro-survival pathways for cancerous cells under the effect of anticancer therapies. Notably, EGFR internalization to the nucleus was also believed to induce resistance to radiotherapy, DNA-damaging agents and EGFR-targeted therapy by different mechanisms (14-17). Recently, another finding has led to the argument that nuclear EGFR upon exposure to stress modulates the stability and translation of microRNA (miRNA)-regulated mRNAs, which enables cells to express proteins directly in response to EGFR activation. This may also contribute to resistance in EGFR-overexpressing tumors (18). In this study, we correlated EGFR mRNA and EGFR protein expression, as well as $E G F R$ gene amplification and mutation with clinical drug response in order to investigate EGFR-mediated resistance.

\section{Materials and Methods}

Cell lines and compounds. The panel of 60 human tumor cell lines of the Developmental Therapeutics Program of the National Cancer Institute (NCI, USA) consisted of leukemia, melanoma, non-small cell lung cancer, colon cancer, renal cancer, ovarian cancer, breast cancer, and prostate carcinoma cells as well as tumor cells of the 
central nervous system. Their origin and processing have been described elsewhere (19). We selected a library of 83 standard anticancer drugs taken from the NCI website (http://dtp.nci.nih.gov), in order to obtain an overview of the relevance of EGFR for all clinically relevant compounds. Cytotoxicity of tested compounds towards cells were assayed by the means of sulforhodamine B (SRB) assay (20). All compounds were tested in the range from $10^{-4}$ to $10^{-8} \mathrm{M}$.

Statistical analysis. Data from mRNA microarrays of the NCI tumor cell line panel $(21,22)$ were sourced through the NCI website (http://dtp.nci.nih.gov). The Pearson correlation test was used to test the correlation of $\log _{10} \mathrm{IC}_{50}$ values with the relative mRNA or protein expression values, as well as the gene copy numbers and the mutational status. This test was implemented using the WinSTAT Program (Kalmia, Cambridge, MA, USA). $p$-Values were calculated for statistical significance, and $r$-values were calculated for the correlation coefficient. Significant positive correlations $(p<0.05$ and $r>0.2$ ) between drug $\log _{10} \mathrm{IC}_{50}$ and EGFR data indicated that EGFR was a factor for resistance to the specific drug, while significant negative correlations $(p<0.05$ and $r<-0.2)$ indicated that EGFR mediated sensitivity to the drug.

\section{Results}

Correlation of $\log _{10} I C_{50}$ for standard anticancer drugs to EGFR expression and mutations. Dose-response curves of a total of 83 standard anticancer agents were used to calculate $\log _{10} \mathrm{IC}_{50}$ values for 60 cell lines of different tumor origin. These values were deposited at the website of the Developmental Therapeutics Program of the NCI (http://dtp.cancer.gov). Data for $E G F R$ mRNA and protein expression, as well as $E G F R$ gene amplification and mutation, were sourced from the same database. We correlated the $\log _{10} \mathrm{IC}_{50}$ values with the EGFR data by Pearson correlation test (Table I). The significant relationship of high $\log _{10} \mathrm{IC}_{50}$ values for busulfan to high EGFR mRNA ( $p=0.019, r=0.276$ ) and protein expression $(p<0.001, r=0.444)$ of tumor cell lines indicated that $E G F R$ expression may represent a factor of resistance to this drug (Table I). However, no significant $p$ values were observed for the correlation between $\log _{10} \mathrm{IC}_{50}$ values for busulfan and EGFR gene copy number or point mutations, indicating that mRNA or protein expression, but not genetic aberrations in the $E G F R$ gene, may affect responsiveness of tumor cells to this drug. The EGFR mRNA expression as determined by microarray expression $(8 / 16=50 \%)$ or RNase protection assay $(11 / 16=69 \%)$ was significantly correlated with alkylating and platinum drugs. Concerning protein expression measured by western blotting, the fraction of drugs with significant correlations was even higher $(13 / 16=81 \%)$. EGFR gene amplification and mutations were less frequently associated with resistance to alkylating or platinum drugs $(2 / 16=13 \%$ and $0 / 16=0 \%$, respectively).

We also investigated the relationships between EGFR expression or genetic aberrations and drugs of other pharmacological classes. Cellular response to alkylating agents/platinum drugs, anthracyclines/DNA topoisomerase (TOPO) II inhibitors, epipodophyllotoxins/TOPO II inhibitors or anti-hormones frequently correlated with EGFR as determined by EGFR RNase protection assay and EGFR western blotting (both frequencies $>50 \%$ ), while cellular response to anti-metabolites, antibiotics and mammalian target of rapamycin (mTOR) inhibitors was less frequently associated with EGFR expression (both frequencies $\leq 50 \%$ ). Moderate correlations were found for drugs from the classes of mitotic spindle poisons, camptothecins/TOPO I inhibitors, tyrosine kinase inhibitors, epigenetic inhibitors and the other drug classes investigated. Interestingly, gene copy number or point mutations were considerably less significantly associated with the response of the tumor cell lines to drugs from all pharmacological classes, indicating that genetic alterations are not reliable determinants of drug resistance in this panel of tumor cell lines. EGFR protein expression rather than other measurements was most frequently associated with drug response $(47 / 83=57 \%)$ compared to $41 \%$ for microarray, $53 \%$ for RNase protection, $16 \%$ for gene copy number and $8 \%$ for gene mutation.

Correlation of $\log _{10} I C_{50}$ for natural products with EGFR expression and mutations. As a further step, we correlated the top 10 cytotoxic natural products from a database of more than 1,000 phytochemicals derived from medicinal plants. The information for these 10 natural products have already been reported by us (23). The mean $\log _{10} \mathrm{IC}_{50}$ values of these 10 compounds across the 60 cell lines of the NCI panel ranged from $-8.263( \pm 0.427)$ to $-12.346( \pm 9.44) \mathrm{M}(23)$. We correlated all $\log _{10} \mathrm{IC}_{50}$ values for the 10 compounds with EGFR data of the cell lines. As shown in Table I, the cytotoxicity of $6 / 10$ compounds was neither associated with EGFR mRNA nor EGFR protein expression, indicating the potential of these natural products in overcoming EGFRmediated resistance to standard anticancer drugs.

\section{Discussion}

EGFR is well-known as oncogenic factor. The development of specific small molecules and antibodies targeting EGFR represents an attractive treatment strategy to eliminate EGFR-overexpressing tumors $(24,25)$. It is also long known from transfection experiments that the EGFR gene and other human epidermal growth factor receptor family members confer resistance to chemo- and radiotherapy (26-28).

Despite these interesting results, the relevance of EGFR as a factor of resistance to multiple drugs remains ambiguous. In the present investigation, we explored whether EGFR data were correlated with a single drug, a specific drug class, or a broad spectrum of drugs, which might be comparable to the multidrug-resistance phenotype mediated by ATP-binding cassette $(\mathrm{ABC})$ transporters. 
Table I. Correlation of the epidermal growth factor receptor (EGFR) mRNA and protein levels to the log-transformed half-maximal inhibitory concentration $\left(\log _{10} I C_{50}\right)$ data for standard anticancer drugs and natural products in the panel of tumor cell lines.

\begin{tabular}{|c|c|c|c|c|c|c|c|}
\hline \multirow[b]{2}{*}{ Drug class } & & \multicolumn{6}{|c|}{$E G F R$} \\
\hline & & $\begin{array}{l}\text { Pearson } \\
\text { correlation }\end{array}$ & $\begin{array}{c}\text { Microarray } \\
\text { hybridization }\end{array}$ & $\begin{array}{c}\text { RNase } \\
\text { protection assay }\end{array}$ & $\begin{array}{l}\text { Western } \\
\text { blotting }\end{array}$ & $\begin{array}{c}\text { Gene copy } \\
\text { number }\end{array}$ & $\begin{array}{c}\text { Point } \\
\text { mutations }\end{array}$ \\
\hline \multirow{38}{*}{$\begin{array}{l}\text { Alkylating agents } \\
\text { and platinum-based } \\
\text { compounds }\end{array}$} & $E G F R$ RNase protection assay & $r$ & 0.727 & & 0.636 & & \\
\hline & & $p$ & $1.09 \times 10^{-10}$ & & $3.98 \times 10^{-8}$ & & \\
\hline & EGFR western blotting & $r$ & 0.609 & 0.636 & & & \\
\hline & & $p$ & $1.99 \times 10^{-7}$ & $3.98 \times 10^{-8}$ & & & \\
\hline & Busulfan & $r$ & 0.276 & 0.339 & 0.444 & 0.090 & -0.032 \\
\hline & & $p$ & 0.019 & 0.005 & $2.41 \times 10^{-4}$ & 0.250 & 0.407 \\
\hline & Dacarbazine & $r$ & 0.263 & 0.300 & 0.287 & 0.123 & $4.64 \times 10^{-4}$ \\
\hline & & $p$ & 0.025 & 0.012 & 0.014 & 0.179 & 0.499 \\
\hline & Thiotepa & $r$ & 0.177 & 0.134 & 0.315 & -0.0336 & 0.150 \\
\hline & & $p$ & 0.092 & 0.157 & 0.007 & 0.340 & 0.128 \\
\hline & Carmustine & $r$ & 0.328 & 0.385 & 0.518 & 0.080 & 0.003 \\
\hline & & $p$ & 0.006 & 0.001 & $1.13 \times 10^{-5}$ & 0.272 & 0.491 \\
\hline & Lomustine & $r$ & 0.057 & 0.237 & 0.102 & 0.260 & 0.056 \\
\hline & & $p$ & 0.336 & 0.038 & 0.221 & 0.023 & 0.338 \\
\hline & Semustine & $r$ & 0.243 & 0.309 & 0.497 & 0.047 & 0.001 \\
\hline & & $p$ & 0.034 & 0.010 & $3.18 \times 10^{-5}$ & 0.363 & 0.496 \\
\hline & Bendamustine & $r$ & 0.045 & 0.222 & 0.287 & -0.012 & 0.108 \\
\hline & & $p$ & 0.368 & 0.048 & 0.014 & 0.464 & 0.209 \\
\hline & Streptozocin & $r$ & -0.052 & 0.148 & 0.322 & -0.024 & -0.013 \\
\hline & & $p$ & 0.352 & 0.136 & 0.006 & 0.430 & 0.463 \\
\hline & Chlorambucil & $r$ & 0.219 & 0.231 & 0.429 & -0.060 & 0.106 \\
\hline & & $p$ & 0.049 & 0.040 & $3.17 \times 10^{-4}$ & 0.326 & 0.213 \\
\hline & Ifosfamide & $r$ & 0.256 & 0.236 & 0.447 & 0.020 & -0.066 \\
\hline & & $p$ & 0.027 & 0.039 & $1.96 \times 10^{-4}$ & 0.439 & 0.311 \\
\hline & Mafosfamide & $r$ & 0.080 & 0.029 & 0.249 & -0.082 & -0.039 \\
\hline & & $p$ & 0.276 & 0.413 & 0.027 & 0.268 & 0.386 \\
\hline & Melphalan & $r$ & 0.297 & 0.343 & 0.446 & 0.048 & 0.133 \\
\hline & & $p$ & 0.012 & 0.005 & $1.98 \times 10^{-4}$ & 0.359 & 0.161 \\
\hline & Azathioprine & $r$ & -0.045 & 0.147 & 0.176 & 0.246 & 0.053 \\
\hline & & $p$ & 0.368 & 0.140 & 0.093 & 0.031 & 0.346 \\
\hline & Carboplatin & $r$ & 0.216 & 0.233 & 0.195 & 0.135 & 0.112 \\
\hline & & $p$ & 0.052 & 0.034 & 0.068 & 0.152 & 0.199 \\
\hline & Cisplatin & $r$ & 0.208 & 0.123 & 0.240 & -0.002 & 0.069 \\
\hline & & $p$ & 0.058 & 0.166 & 0.032 & 0.495 & 0.302 \\
\hline & Oxaliplatin & $r$ & 0.220 & 0.253 & 0.345 & 0.209 & -0.130 \\
\hline & & $p$ & 0.048 & 0.029 & 0.004 & 0.056 & 0.163 \\
\hline & Resistant, $\mathrm{n} / \mathrm{N}$ & & $8 / 16(50 \%)$ & $11 / 16(69 \%)$ & $13 / 16(81 \%)$ & $2 / 16(13 \%)$ & $0 / 16(0 \%)$ \\
\hline & Sensitive, $\mathrm{n} / \mathrm{N}$ & & $0 / 16(0 \%)$ & $0 / 16(0 \%)$ & $0 / 16(0 \%)$ & $0 / 16(0 \%)$ & $0 / 16(0 \%)$ \\
\hline \multirow[t]{16}{*}{ Anti-metabolites } & Methotrexate & $r$ & 0.201 & 0.195 & 0.191 & 0.066 & 0.072 \\
\hline & & $p$ & 0.065 & 0.071 & 0.072 & 0.307 & 0.295 \\
\hline & Trimetrexate & $r$ & 0.298 & 0.248 & 0.267 & 0.064 & -0.012 \\
\hline & & $p$ & 0.012 & 0.030 & 0.020 & 0.314 & 0.464 \\
\hline & Pemetrexed & $r$ & -0.092 & -0.024 & 0.123 & 0.038 & 0.078 \\
\hline & & $p$ & 0.250 & 0.430 & 0.178 & 0.388 & 0.281 \\
\hline & Hydroxyurea & $r$ & 0.003 & 0.137 & 0.262 & -0.013 & 0.083 \\
\hline & & $p$ & 0.491 & 0.155 & 0.023 & 0.462 & 0.268 \\
\hline & Cladribine & $r$ & -0.062 & 0.005 & 0.114 & 0.043 & 0.202 \\
\hline & & $p$ & 0.321 & 0.485 & 0.195 & 0.374 & 0.063 \\
\hline & Clofarabine & $r$ & -0.121 & -0.073 & 0.072 & 0.082 & 0.229 \\
\hline & & $p$ & 0.184 & 0.294 & 0.294 & 0.268 & 0.041 \\
\hline & Fludarabine & $r$ & -0.041 & 0.059 & 0.007 & 0.050 & 0.049 \\
\hline & & $p$ & 0.383 & 0.333 & 0.480 & 0.354 & 0.359 \\
\hline & Mercaptopurine & $r$ & 0.180 & 0.253 & 0.180 & 0.311 & 0.311 \\
\hline & & $p$ & 0.088 & 0.028 & 0.084 & 0.008 & 0.008 \\
\hline
\end{tabular}


Table I. Continued

\begin{tabular}{|c|c|c|c|c|c|c|c|}
\hline \multirow[b]{2}{*}{ Drug class } & & \multicolumn{6}{|c|}{$E G F R$} \\
\hline & & $\begin{array}{l}\text { Pearson } \\
\text { correlation }\end{array}$ & $\begin{array}{c}\text { Microarray } \\
\text { hybridization }\end{array}$ & $\begin{array}{c}\text { RNase } \\
\text { protection assay }\end{array}$ & $\begin{array}{l}\text { Western } \\
\text { blotting }\end{array}$ & $\begin{array}{c}\text { Gene copy } \\
\text { number }\end{array}$ & $\begin{array}{c}\text { Point } \\
\text { mutations }\end{array}$ \\
\hline & \multirow[t]{2}{*}{ Pentostatin } & $r$ & 0.078 & 0.010 & 0.236 & -0.065 & 0.088 \\
\hline & & $p$ & 0.283 & 0.469 & 0.036 & 0.313 & 0.257 \\
\hline & \multirow[t]{2}{*}{ 6-Thioguanine } & $r$ & 0.202 & 0.200 & 0.169 & 0.188 & 0.181 \\
\hline & & $p$ & 0.064 & 0.066 & 0.098 & 0.075 & 0.085 \\
\hline & \multirow[t]{2}{*}{ Cytarabine } & $r$ & 0.098 & 0.163 & 0.207 & 0.146 & 0.134 \\
\hline & & $p$ & 0.231 & 0.111 & 0.056 & 0.132 & 0.156 \\
\hline & \multirow[t]{2}{*}{ 5-Fluorouracil } & $r$ & 0.170 & 0.168 & 0.139 & 0.070 & -0.002 \\
\hline & & $p$ & 0.100 & 0.103 & 0.145 & 0.297 & 0.495 \\
\hline & \multirow[t]{2}{*}{ Gemcitabine } & $r$ & 0.035 & 0.046 & 0.228 & 0.064 & 0.161 \\
\hline & & $p$ & 0.397 & 0.366 & 0.040 & 0.313 & 0.111 \\
\hline & \multirow{2}{*}{ Tegafur } & $r$ & 0.225 & 0.178 & 0.063 & -0.027 & -0.071 \\
\hline & & $p$ & 0.047 & 0.093 & 0.317 & 0.418 & 0.299 \\
\hline & Resistant, $\mathrm{n} / \mathrm{N}$ & & $2 / 14(14 \%)$ & $2 / 14(14 \%)$ & $4 / 14(29 \%)$ & $1 / 14(7 \%)$ & $2 / 14(14 \%)$ \\
\hline & Sensitive, $\mathrm{n} / \mathrm{N}$ & & $0 / 14(0 \%)$ & $0 / 14(0 \%)$ & $0 / 14(0 \%)$ & $0 / 14(0 \%)$ & $0 / 14(0 \%)$ \\
\hline \multirow{10}{*}{$\begin{array}{l}\text { Mitotic spindle } \\
\text { poisons }\end{array}$} & \multirow[t]{2}{*}{ Docetaxel } & $r$ & 0.206 & 0.270 & 0.075 & 0.197 & 0.029 \\
\hline & & $p$ & 0.064 & 0.022 & 0.287 & 0.069 & 0.414 \\
\hline & \multirow[t]{2}{*}{ Paclitaxel } & $r$ & 0.368 & 0.306 & 0.229 & 0.107 & 0.096 \\
\hline & & $p$ & 0.002 & 0.010 & 0.039 & 0.208 & 0.234 \\
\hline & \multirow[t]{2}{*}{ Vinblastine } & $r$ & 0.286 & 0.344 & 0.208 & 0.183 & -0.018 \\
\hline & & $p$ & 0.015 & 0.004 & 0.056 & 0.081 & 0.446 \\
\hline & \multirow[t]{2}{*}{ Vincristine } & $r$ & 0.106 & 0.151 & 0.115 & 0.115 & 0.149 \\
\hline & & $p$ & 0.215 & 0.129 & 0.192 & 0.191 & 0.131 \\
\hline & Resistant, $\mathrm{n} / \mathrm{N}$ & & $2 / 4(50 \%)$ & $3 / 4(75 \%)$ & $1 / 4(25 \%)$ & $0 / 5(0 \%)$ & $0 / 5(0 \%)$ \\
\hline & Sensitive, $\mathrm{n} / \mathrm{N}$ & & $0 / 4(0 \%)$ & $0 / 4(0 \%)$ & $0 / 4(0 \%)$ & $0 / 4(0 \%)$ & $0 / 4(0 \%)$ \\
\hline \multirow{12}{*}{$\begin{array}{l}\text { Anthracyclines/ } \\
\text { TOPO II inhibitors }\end{array}$} & \multirow[t]{2}{*}{ Daunorubicin } & $r$ & 0.300 & 0.312 & 0.376 & -0.009 & 0.082 \\
\hline & & $p$ & 0.017 & 0.015 & 0.003 & 0.476 & 0.284 \\
\hline & Doxorubicin & $r$ & 0.335 & 0.361 & 0.448 & 0.003 & 0.027 \\
\hline & & $p$ & 0.005 & 0.003 & $1.68 \times 10^{-4}$ & 0.492 & 0.419 \\
\hline & Epirubicin & $r$ & 0.283 & 0.334 & 0.370 & -0.003 & 0.038 \\
\hline & & $p$ & 0.016 & 0.006 & 0.002 & 0.492 & 0.387 \\
\hline & Idarubicin & $r$ & 0.251 & 0.225 & 0.351 & 0.049 & 0.112 \\
\hline & & $p$ & 0.031 & 0.048 & 0.003 & 0.358 & 0.204 \\
\hline & Mitoxantrone & $r$ & 0.051 & 0.015 & 0.258 & -0.112 & 0.119 \\
\hline & & $p$ & 0.353 & 0.454 & 0.023 & 0.197 & 0.185 \\
\hline & Resistant, $\mathrm{n} / \mathrm{N}$ & & $4 / 5(80 \%)$ & $4 / 5(80 \%)$ & $5 / 5(100 \%)$ & $0 / 5(0 \%)$ & $0 / 5(0 \%)$ \\
\hline & Sensitive, $\mathrm{n} / \mathrm{N}$ & & $0 / 5(0 \%)$ & $0 / 5(0 \%)$ & $0 / 5(0 \%)$ & $0 / 5(0 \%)$ & $0 / 5(0 \%)$ \\
\hline Antibiotics & Bleomycin & $r$ & -0.084 & -0.126 & 0.098 & -0.216 & 0.213 \\
\hline & & $p$ & 0.266 & 0.172 & 0.228 & 0.048 & 0.052 \\
\hline & Dactinomycin & $r$ & 0.448 & 0.425 & 0.503 & 0.089 & -0.090 \\
\hline & & $p$ & $4.95 \times 10^{-4}$ & 0.001 & $7.19 \times 10^{-5}$ & 0.266 & 0.263 \\
\hline & Mitomycin C & $r$ & 0.233 & 0.124 & 0.283 & -0.107 & 0.144 \\
\hline & & $p$ & 0.040 & 0.176 & 0.014 & 0.208 & 0.138 \\
\hline & Mithramycin & $r$ & 0.426 & 0.167 & 0.160 & -0.0209 & -0.062 \\
\hline & & $p$ & $5.90 \times 10^{-4}$ & 0.111 & 0.117 & 0.439 & 0.325 \\
\hline & Resistant, $\mathrm{n} / \mathrm{N}$ & & $3 / 4(75 \%)$ & $1 / 4(25 \%)$ & $2 / 4(50 \%)$ & $0 / 4(0 \%)$ & $0 / 4(0 \%)$ \\
\hline & Sensitive, $\mathrm{n} / \mathrm{N}$ & & $0 / 4(0 \%)$ & $0 / 4(0 \%)$ & $0 / 4(0 \%)$ & $1 / 4(25 \%)$ & $0 / 4(0 \%)$ \\
\hline Epipodophyllotoxins/ & Etoposide & $r$ & 0.190 & 0.313 & 0.322 & 0.087 & 0.061 \\
\hline TOPO II inhibitors & & $p$ & 0.080 & 0.009 & 0.007 & 0.259 & 0.325 \\
\hline & Teniposide & $r$ & 0.262 & 0.294 & 0.476 & 0.031 & 0.076 \\
\hline & & $p$ & 0.025 & 0.013 & $7.01 \times 10^{-5}$ & 0.407 & 0.285 \\
\hline & m-AMSA, amsacrine & $r$ & 0.115 & 0.097 & 0.242 & -0.086 & 0.127 \\
\hline & & $p$ & 0.195 & 0.233 & 0.031 & 0.256 & 0.169 \\
\hline & Resistant, $\mathrm{n} / \mathrm{N}$ & & $1 / 3(33 \%)$ & $2 / 3(66 \%)$ & $3 / 3(100 \%)$ & $0 / 4(0 \%)$ & $0 / 4(0 \%)$ \\
\hline & Sensitive, $\mathrm{n} / \mathrm{N}$ & & $0 / 3(0 \%)$ & $0 / 3(0 \%)$ & $0 / 3(0 \%)$ & $0 / 3(0 \%)$ & $0 / 3(0 \%)$ \\
\hline Camptothecins/ & Camptothecin & $r$ & 0.089 & 0.066 & 0.374 & -0.084 & 0.191 \\
\hline TOPO I inhibitors & & $p$ & 0.268 & 0.324 & 0.003 & 0.277 & 0.089 \\
\hline
\end{tabular}


Table I. Continued

\begin{tabular}{|c|c|c|c|c|c|c|c|}
\hline \multirow[b]{2}{*}{ Drug class } & & \multicolumn{6}{|c|}{$E G F R$} \\
\hline & & $\begin{array}{l}\text { Pearson } \\
\text { correlation }\end{array}$ & $\begin{array}{c}\text { Microarray } \\
\text { hybridization }\end{array}$ & $\begin{array}{c}\text { RNase } \\
\text { protection assay }\end{array}$ & $\begin{array}{l}\text { Western } \\
\text { blotting }\end{array}$ & $\begin{array}{l}\text { Gene copy } \\
\text { number }\end{array}$ & $\begin{array}{c}\text { Point } \\
\text { mutations }\end{array}$ \\
\hline \multirow{22}{*}{ Antihormones } & Irinotecan & $r$ & 0.078 & 0.281 & 0.201 & -0.091 & 0.011 \\
\hline & & $p$ & 0.281 & 0.018 & 0.066 & 0.250 & 0.467 \\
\hline & Topotecan & $r$ & 0.148 & 0.067 & 0.308 & -0.023 & 0.244 \\
\hline & & $p$ & 0.134 & 0.309 & 0.008 & 0.431 & 0.031 \\
\hline & Resistant, $\mathrm{n} / \mathrm{N}$ & & $0 / 3(0 \%)$ & $1 / 3(33 \%)$ & $2 / 3(66 \%)$ & $0 / 3(0 \%)$ & $1 / 3(33 \%)$ \\
\hline & Sensitive, $n / N$ & & $0 / 3(0 \%)$ & $0 / 3(0 \%)$ & $0 / 3(0 \%)$ & $0 / 3(0 \%)$ & $0 / 3(0 \%)$ \\
\hline & Fulvestrant & $r$ & 0.199 & 0.332 & 0.361 & 0.162 & -0.140 \\
\hline & & $p$ & 0.068 & 0.006 & 0.003 & 0.113 & 0.147 \\
\hline & Tamoxifen & $r$ & 0.349 & 0.513 & 0.545 & 0.226 & -0.042 \\
\hline & & $p$ & 0.005 & $2.27 \times 10^{-5}$ & $4.01 \times 10^{-6}$ & 0.042 & 0.377 \\
\hline & Toremifen & $r$ & 0.117 & 0.299 & 0.381 & 0.091 & -0.085 \\
\hline & & $p$ & 0.199 & 0.015 & 0.002 & 0.254 & 0.270 \\
\hline & Anastrozol & $r$ & -0.128 & 0.073 & -0.044 & -0.058 & 0.006 \\
\hline & & $p$ & 0.191 & 0.314 & 0.382 & 0.346 & 0.484 \\
\hline & Exemestane & $r$ & 0.298 & 0.237 & 0.068 & 0.026 & 0.055 \\
\hline & & $p$ & 0.011 & 0.039 & 0.307 & 0.425 & 0.342 \\
\hline & Megostrol & $r$ & 0.036 & 0.202 & 0.239 & -0.049 & -0.237 \\
\hline & & $p$ & 0.396 & 0.068 & 0.036 & 0.357 & 0.037 \\
\hline & Raloxifene & $r$ & 0.293 & 0.535 & 0.500 & 0.288 & -0.119 \\
\hline & & $p$ & 0.013 & $0.15 \times 10^{-6}$ & $2.81 \times 10^{-5}$ & 0.013 & 0.184 \\
\hline & Resistant, $\mathrm{n} / \mathrm{N}$ & & $3 / 7(43 \%)$ & $5 / 7(71 \%)$ & $5 / 7(71 \%)$ & $2 / 7(29 \%)$ & $0 / 7(0 \%)$ \\
\hline & Sensitive, $n / N$ & & $0 / 7(0 \%)$ & $0 / 7(0 \%)$ & $0 / 7(0 \%)$ & $0 / 7(0 \%)$ & $1 / 7(14 \%)$ \\
\hline \multirow{30}{*}{$\begin{array}{l}\text { Tyrosine kinase } \\
\text { inhibitors }\end{array}$} & Axitinib & $r$ & -0.151 & 0.088 & 0.155 & -0.028 & -0.011 \\
\hline & & $p$ & 0.145 & 0.271 & 0.136 & 0.423 & 0.470 \\
\hline & Crizotinib & $r$ & 0.344 & 0.464 & 0.408 & 0.151 & 0.030 \\
\hline & & $p$ & 0.004 & $1.41 \times 10^{-4}$ & $6.79 \times 10^{-4}$ & 0.127 & 0.412 \\
\hline & Dasatinib & $r$ & -0.284 & -0.489 & -0.237 & -0.126 & 0.206 \\
\hline & & $p$ & 0.021 & $1.34 \times 10^{-4}$ & 0.043 & 0.184 & 0.069 \\
\hline & Erlotinib & $r$ & -0.356 & -0.450 & -0.326 & -0.245 & 0.164 \\
\hline & & $p$ & 0.003 & $1.94 \times 10^{-4}$ & 0.006 & 0.029 & 0.107 \\
\hline & Gamitrinib G-TPP & $r$ & 0.442 & 0.503 & 0.315 & 0.060 & -0.150 \\
\hline & & $p$ & $2.56 \times 10^{-4}$ & $3.28 \times 10^{-5}$ & 0.008 & 0.327 & 0.129 \\
\hline & Gamitrinib G4 & $r$ & 0.487 & 0.341 & 0.282 & 0.118 & -0.044 \\
\hline & & $p$ & $5.20 \times 10^{-5}$ & 0.005 & 0.015 & 0.187 & 0.370 \\
\hline & Gefitinib & $r$ & -0.090 & -0.128 & -0.124 & -0.132 & -0.236 \\
\hline & & $p$ & 0.250 & 0.173 & 0.174 & 0.159 & 0.036 \\
\hline & Imatinib & $r$ & 0.181 & 0.343 & 0.263 & 0.181 & -0.074 \\
\hline & & $p$ & 0.099 & 0.007 & 0.029 & 0.097 & 0.299 \\
\hline & Lapatinib & $r$ & 0.206 & -0.058 & -0.023 & 0.086 & 0.006 \\
\hline & & $p$ & 0.071 & 0.342 & 0.436 & 0.271 & 0.483 \\
\hline & Nilotinib & $r$ & 0.242 & 0.342 & 0.205 & 0.430 & -0.071 \\
\hline & & $p$ & 0.042 & 0.007 & 0.071 & $6.67 \times 10^{-4}$ & 0.306 \\
\hline & Sorafenib & $r$ & 0.201 & 0.290 & 0.207 & 0.056 & -0.317 \\
\hline & & $p$ & 0.077 & 0.019 & 0.068 & 0.344 & 0.010 \\
\hline & Sunitinib & $r$ & 0.129 & 0.267 & 0.143 & 0.120 & -0.070 \\
\hline & & $p$ & 0.168 & 0.022 & 0.140 & 0.182 & 0.300 \\
\hline & Vandetanib & $r$ & -0.204 & -0.193 & -0.070 & -0.240 & 0.007 \\
\hline & & $p$ & 0.066 & 0.079 & 0.302 & 0.036 & 0.481 \\
\hline & Vemurafenib & $r$ & 0.303 & 0.29 & 0.153 & 0.163 & -0.274 \\
\hline & & $p$ & 0.010 & 0.014 & 0.124 & 0.108 & 0.018 \\
\hline & Resistant, $\mathrm{n} / \mathrm{N}$ & & $5 / 14(36 \%)$ & $8 / 14(57 \%)$ & $4 / 14(29 \%)$ & $1 / 14(7 \%)$ & $0 / 14(0 \%)$ \\
\hline & Sensitive, $\mathrm{n} / \mathrm{N}$ & & $2 / 14(14 \%)$ & $2 / 14(14 \%)$ & $2 / 14(14 \%)$ & $2 / 14(14 \%)$ & $3 / 14(21 \%)$ \\
\hline \multirow[t]{3}{*}{ mTOR inhibitors } & Everolimus & $r$ & -0.190 & -0.169 & -0.185 & -0.139 & 0.168 \\
\hline & & $p$ & 0.076 & 0.104 & 0.080 & 0.147 & 0.101 \\
\hline & Temsirolimus & $r$ & 0.081 & 0.058 & 0.107 & -0.133 & -0.027 \\
\hline
\end{tabular}


Table I. Continued

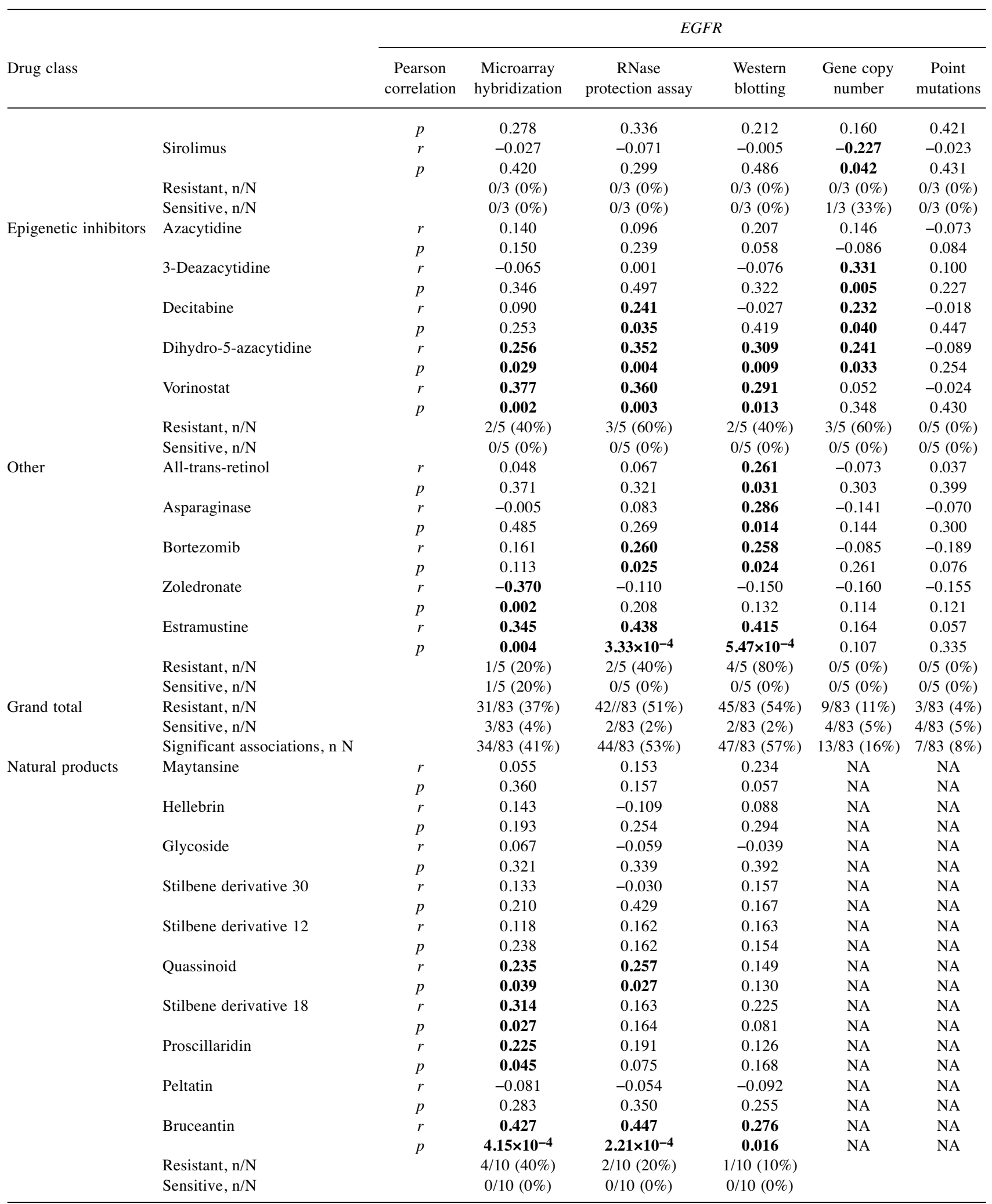

Bold numbers: Significant associations of between $\log _{10} \mathrm{IC}_{50}$ and EGFR $(p<0.05) . r>0.2$ demonstrated a positive association which implied EGFR mediated resistance and vice versa. 
Our results showed that the expression of EGFR mRNA or EGFR protein was mainly correlated with the $\log _{10} \mathrm{IC}_{50}$ of anti-hormones and inducers of DNA damage, e.g. alkylating agents/platinum drugs, anthracyclines/TOPO II inhibitors, epipodophyllotoxins/TOPO II inhibitors, but rarely with antimetabolites, antibiotics and mTOR inhibitors. By comparison, cross-resistance mediated by P-glycoprotein comprises the drug classes of anthracyclines/ TOPO II inhibitors, mitotic spindle poisons and some antibiotics, but not alkylating agents/platinum drugs or anti-hormones (29). Hence crossresistance mediated by P-glycoprotein and EGFR exhibits overlapping but not identical profiles. It has been reported that EGFR regulates resistance to various modes of cell death (e.g. apoptosis, autophagy, and anoikis) (30-32). The suppression of cell death represents an imperative step in carcinogenesis but also in resistance to chemotherapy. EGFR protein expression was shown to mediate resistance to cisplatin in the clinic by Aichler et al. (33). Sensitivity to erlotinib was reported in a clinical trial by Juergens et al. (34) and in preclinical studies by Quesnelle et al. (35). These results are consistent with our in vitro results with the NCI60 cell line panel.

Another result of our study was that drug resistance of the NCI panel of cell lines correlated with EGFR mRNA and protein expression but not with gene copy number or gene mutations. This may be surprising at first sight, since many investigations reported that EGFR gene amplification and mutations are associated with treatment failure and poor prognosis (36-39). However, comparable results were observed by others for the NCI cell line panel showing that response did not correlate with $E G F R$ amplification but with $E G F R$ gene expression (40). Still, it cannot be excluded that the current panel of tumor cell lines, which consists of diverse tumor types (carcinoma of colon, lung, kidney, prostate, ovarian and breast, CNS tumors, melanoma, leukemia) was too heterogeneous to allow elucidation of significant relationships between the mutational status and drug resistance.

In addition to standard anticancer drugs, we investigated phytochemicals. Previously, we described a library of more than 1,000 chemically characterized compounds derived from medicinal plants used in Chinese medicine (23). We tested correlations of the $\log _{10} \mathrm{IC}_{50}$ for the 10 most cytotoxic natural products and the EGFR parameters and found that for the majority of these phytochemicals $(6 / 10)$, there was no significant correlation with any of the EGFR data. Such compounds can be further developed as drugs with activity against EGFR-overexpressing or mutated tumors, as they were not part of the EGFR-associated cross-resistance profile.

In conclusion, our study revealed the association between EGFR and resistance to a broad range of clinical drugs. EGFR is not only a factor in carcinogenesis, but probably also plays an essential role in development of drug resistance.

\section{Conflicts of Interest}

The Authors declare that there is no conflict of interest.

\section{Authors' Contributions}

Ge Yan contributed to article composing. Dr. Thomas Efferth contributed to the data generation and revision.

\section{Acknowledgements}

The Authors thank the Chinese Scholarship Council (Beijing, R.P. China) for Ph.D. program stipend to G. Yan.

\section{References}

1 Mitsudomi T and Yatabe Y: Epidermal growth factor receptor in relation to tumor development. EGFR gene and cancer. FEBS J 277: 301-308, 2010. PMID: 19922469. DOI: 10.1111/j.17424658.2009.07448.x

2 Juchum M, Günther M and Laufer SA: Fighting cancer drug resistance. Opportunities and challenges for mutation-specific EGFR inhibitors. Drug Resist Updat 20: 12-28, 2015. PMID: 26021435. DOI: $10.1016 /$ j.drup.2015.05.002

3 Wang S, Song Y, Yan F and Liu D: Mechanisms of resistance to third-generation EGFR tyrosine kinase inhibitors. Front Med 10: 383388, 2016. PMID: 27770386. DOI: 10.1007/s11684-016-0488-1

4 Wu S-G and Shih J-Y: Management of acquired resistance to EGFR TKI-targeted therapy in advanced non-small cell lung cancer. Molecular Cancer 17: 38, 2018. PMID: 29455650. DOI: 10.1186/s12943-018-0777-1

5 Harari PM: Epidermal growth factor receptor inhibition strategies in oncology. Endocrine-Related Cancer 11: 689-708, 2004. PMID: 15613446. DOI: 10.1677/erc.1.00600

6 Martinelli E, Palma R de, Orditura M, Vita F de and Ciardiello F: Anti-epidermal growth factor receptor monoclonal antibodies in cancer therapy. Clin Exp Immunol 158: 1-9, 2009. PMID: 19737224. DOI: $10.1111 / \mathrm{j} .1365-2249.2009 .03992 . x$

7 Reddy LH and Couvreur P, eds.: Macromolecular Anticancer Therapeutics. Springer-Verlag, New York, NY. 2010.

8 Mazorra Z, Lavastida A, Concha-Benavente F, Valdés A, Srivastava RM, García-Bates TM, Hechavarría E, González Z, González A, Lugiollo M, Cuevas I, Frómeta C, Mestre BF, Barroso MC, Crombet T and Ferris RL: Nimotuzumab induces NK cell activation, cytotoxicity, dendritic cell maturation and expansion of EGFR-specific T-cells in head and neck cancer patients. Front Pharmacol 8: 382, 2017. PMID: 28674498. DOI: 10.3389/fphar.2017.00382

9 Kol A, van Terwisscha Scheltinga A, Pool M, Gerdes C, Vries $\mathrm{E}$ de and Jong S de: ADCC responses and blocking of EGFRmediated signaling and cell growth by combining the anti-EGFR antibodies imgatuzumab and cetuximab in NSCLC cells. Oncotarget 8: 45432-45446, 2017. PMID: 28467975. DOI: 10.18632/oncotarget.17139

10 Topper MB, Tonra JR, Pytowski B and Eastman SW: Differentiation between the EGFR antibodies necitumumab, cetuximab, and panitumumab. Antibody internalization and EGFR degradation. J Clin Oncol 29: e13022-e13022, 2011. DOI: 10.1200/jco.2011.29.15_suppl.e13022 
11 Fitzgerald TL, Lertpiriyapong K, Cocco L, Martelli AM, Libra M, Candido S, Montalto G, Cervello M, Steelman L, Abrams SL and McCubrey JA: Roles of EGFR and KRAS and their downstream signaling pathways in pancreatic cancer and pancreatic cancer stem cells. Adv Biol Regul 59: 65-81, 2015. PMID: 26257206. DOI: 10.1016/j.jbior.2015.06.003

12 Lo H-W: EGFR-targeted therapy in malignant glioma. Novel aspects and mechanisms of drug resistance. Curr Mol Pharmacol 3: 37-52, 2010. PMID: 20030624.

13 Zhu H, Cao X, Ali-Osman F, Keir S and Lo H-W: EGFR and EGFRvIII interact with PUMA to inhibit mitochondrial translocalization of PUMA and PUMA-mediated apoptosis independent of EGFR kinase activity. Cancer Lett 294: 101-110, 2010. PMID: 20153921. DOI: 10.1016/j.canlet.2010.01.028

14 Lo H-W: Nuclear mode of the EGFR signaling network. Biology, prognostic value, and therapeutic implications. Discov Med 10: 44-51, 2010. PMID: 20670598.

15 Lo H-W and Hung M-C: Nuclear EGFR signalling network in cancers. Linking EGFR pathway to cell cycle progression, nitric oxide pathway and patient survival. Br J Cancer 94: 184-188, 2006. PMID: 16434982. DOI: 10.1038/sj.bjc.6602941

16 Huang W-C, Chen Y-J and Hung M-C: Implication of nuclear EGFR in the development of resistance to anticancer therapies. BioMedicine 1: 2-10, 2011. DOI: 10.1016/j.biomed. 2011.10.001

17 Li C, Iida M, Dunn EF, Ghia AJ and Wheeler DL: Nuclear EGFR contributes to acquired resistance to cetuximab. Oncogene 28: 3801-3813, 2009. PMID: 19684613. DOI: 10.1038/onc.2009.234

18 Dittmann K, Mayer C, Czemmel S, Huber SM and Rodemann HP: New roles for nuclear EGFR in regulating the stability and translation of mRNAs associated with VEGF signaling. PloS One 12: e0189087, 2017. PMID: 29253018. DOI: 10.1371/ journal.pone. 0189087

19 Alley MC, Scudiero DA, Monks A, Hursey ML, Czerwinski MJ, Fine DL, Abbott BJ, Mayo JG, Shoemaker RH and Boyd MR: Feasibility of drug screening with panels of human tumor cell lines using a microculture tetrazolium assay. Cancer Res 48: 589-601, 1988. PMID: 3335022.

20 Rubinstein LV, Shoemaker RH, Paull KD, Simon RM, Tosini S, Skehan P, Scudiero DA, Monks A and Boyd MR: Comparison of in vitro anticancer-drug-screening data generated with a tetrazolium assay versus a protein assay against a diverse panel of human tumor cell lines. J Natl Cancer Inst 82: 1113-1118, 1990. PMID: 2359137.

21 Scherf U, Ross DT, Waltham M, Smith LH, Lee JK, Tanabe L, Kohn KW, Reinhold WC, Myers TG, Andrews DT, Scudiero DA, Eisen MB, Sausville EA, Pommier Y, Botstein D, Brown PO and Weinstein JN: A gene expression database for the molecular pharmacology of cancer. Nat Genet 24: 236-244, 2000PMID: 10700175. DOI: 10.1038/73439

22 Staunton JE, Slonim DK, Coller HA, Tamayo P, Angelo MJ, Park J, Scherf U, Lee JK, Reinhold WO, Weinstein JN, Mesirov JP, Lander ES and Golub TR: Chemosensitivity prediction by transcriptional profiling. Proc Natl Acad Sci USA 98: 10787-10792, 2001. PMID: 11553813. DOI: $10.1073 /$ pnas. 191368598

23 Konkimalla VB and Efferth T: Anticancer natural product library from traditional Chinese medicine. Comb Chem High Throughput Screen 11: 7-15, 2008. PMID: 18220539.
24 Efferth T: Signal transduction pathways of the epidermal growth factor receptor in colorectal cancer and their inhibition by small molecules. Curr Med Chem 19: 5735-5744, 2012. PMID: 23033949.

25 Kadioglu O, Cao J, Saeed MEM, Greten HJ and Efferth T: Targeting epidermal growth factor receptors and downstream signaling pathways in cancer by phytochemicals. Targeted Oncol 10: 337-353, 2015. PMID: 25410594. DOI: 10.1007/s 11523014-0339-4

26 Kim R, Tanabe K, Uchida Y, Osaki A and Toge T: The role of HER-2 oncoprotein in drug-sensitivity in breast cancer (review). Oncol Rep 9: 3-9, 2002. PMID: 11748447.

27 Navolanic PM, Steelman LS and McCubrey JA: EGFR family signaling and its association with breast cancer development and resistance to chemotherapy (Review). Int J Oncol 22: 237-252, 2003. PMID: 12527919.

28 Schmidt-Ullrich RK, Contessa JN, Lammering G, Amorino G and Lin P-S: ERBB receptor tyrosine kinases and cellular radiation responses. Oncogene 22: 5855-5865, 2003. PMID: 12947392. DOI: $10.1038 /$ sj.onc. 1206698

29 Efferth T, Konkimalla VB, Wang Y-F, Sauerbrey A, Meinhardt S, Zintl F, Mattern J and Volm M: Prediction of broad spectrum resistance of tumors towards anticancer drugs. Clin Cancer Res 14: 2405-2412, 2008. PMID: 18413831. DOI: 10.1158/10780432.CCR-07-4525

30 Jimeno A and Hidalgo M: Blockade of epidermal growth factor receptor (EGFR) activity. Crit Rev Oncol Hematol 53: 179-192, 2005. PMID: 15718144. DOI: 10.1016/j.critrevonc.2004.10.005

31 Jutten B and Rouschop KMA: EGFR signaling and autophagy dependence for growth, survival, and therapy resistance. Cell Cycle 13: 42-51, 2014. PMID: 24335351. DOI: 10.4161/ cc. 27518

32 Kim H, Sung JY, Park E-K, Kho S, Koo KH, Park S-Y, Goh S$\mathrm{H}$, Jeon YK, Oh S, Park B-K, Jung Y-K and Kim Y-N: Regulation of anoikis resistance by NADPH oxidase 4 and epidermal growth factor receptor. Br J Cancer 116: 370-381, 2017. PMID: 28081539. DOI: 10.1038/bjc.2016.440

33 Aichler M, Motschmann M, Jütting U, Luber B, Becker K, Ott K, Lordick F, Langer R, Feith M, Siewert JR and Walch A: Epidermal growth factor receptor (EGFR) is an independent adverse prognostic factor in esophageal adenocarcinoma patients treated with cisplatin-based neoadjuvant chemotherapy. Oncotarget 5: 6620-6632, 2014. PMID: 25216514. DOI: 10.18632/oncotarget. 2268

34 Juergens RA, Bratman SV, Tsao M-S, Laurie SA, Sara Kuruvilla M, Razak ARA and Hansen AR: Biology and patterns of response to EGFR-inhibition in squamous cell cancers of the lung and head and neck. Cancer Treatm Rev 54: 43-57, 2017. PMID: 28192747. DOI: 10.1016/j.ctrv.2017.01.003

35 Quesnelle KM, Wheeler SE, Ratay MK and Grandis JR: Preclinical modeling of EGFR inhibitor resistance in head and neck cancer. Cancer Biol Ther 13: 935-945, 2012. PMID: 22785204. DOI: $10.4161 / \mathrm{cbt} .20846$

36 Park HS, Jang MH, Kim EJ, Kim HJ, Lee HJ, Kim YJ, Kim JH, Kang E, Kim S-W, Kim IA and Park SY: High EGFR gene copy number predicts poor outcome in triple-negative breast cancer. Mod Pathol 27: 1212-1222, 2014. PMID: 24406864. DOI: 10.1038/modpathol.2013.251

37 Woelber L, Hess S, Bohlken H, Tennstedt P, Eulenburg C, Simon R, Gieseking F, Jaenicke F, Mahner S and Choschzick M: EGFR 
gene copy number increase in vulvar carcinomas is linked with poor clinical outcome. J Clin Pathol 65: 133-139, 2012. PMID: 22128196. DOI: $10.1136 / \mathrm{jcp}-2010-079806$

38 Lam DCL, Tam TCC, Lau KMK, Wong W-M, Hui CKM, Lam JCM, Wang JKL, Lui MMS, Ho JCM and Ip MSM: Plasma EGFR mutation detection associated with survival outcomes in advanced-stage lung cancer. Clin Lung Cancer 16: 507-513, 2015. PMID: 26239567. DOI: 10.1016/j.cllc.2015.06.003

39 Kobayashi S, Boggon TJ, Dayaram T, Jänne PA, Kocher O, Meyerson M, Johnson BE, Eck MJ, Tenen DG and Halmos B: $E G F R$ mutation and resistance of non-small-cell lung cancer to gefitinib. N Engl J Med 352: 786-792, 2005. PMID: 15728811. DOI: $10.1056 /$ NEJMoa044238
40 Liu W, Wu X, Zhang W, Montenegro RC, Fackenthal DL, Spitz JA, Huff LM, Innocenti F, Das S, Cook EH, Cox NJ, Bates SE and Ratain MJ: Relationship of EGFR mutations, expression, amplification, and polymorphisms to epidermal growth factor receptor inhibitors in the NCI60 cell lines. Clin Cancer Res 13: 6788-6795, 2007. PMID: 18006781. DOI: 10.1158/10780432.CCR-07-0547

Received April 18, 2019

Revised May 23, 2019

Accepted May 27, 2019 